\title{
CONTROL QUÍMICO DE DIAPHORINA CITRI (KUWAYAMA) (HEMIPTERA: LIVIIDAE) EN LIMA PERSA
}

\author{
ISABEL RUIZ-GALVÁN ${ }^{1}$, NÉSTOR BAUTISTA-MARTÍNEZ ${ }^{1}$, HuSSEIN SÁNCHEZ-ARROYO${ }^{1}$ \\ y Fernando Alberto VALENZUELA ESCOBOZA ${ }^{2}$
}

\author{
${ }^{1}$ Posgrado en Fitosanidad, Colegio de Postgraduados, Campus Montecillo, Km. 36.5 Carretera México-Texcoco, \\ Montecillo, Estado de México C. P. 56230. \\ ${ }^{2}$ Escuela Superior de Agricultura del Valle del Fuerte, Universidad Autónoma de Sinaloa. Calle 16 Av. Japaraqui \\ S/N C.P. 81110. Juan José Ríos, Ahome, Sinaloa, México. <nestor@colpos.mx> \\ Recibido: 07/08/2013; aceptado: 20/08/2014
}

\begin{abstract}
Ruiz-Galván, I., Bautista-Martínez, N., Sánchez-Arroyo, H. \& Valenzuela Escoboza, F. A. 2015. Control químico de Diaphorina citri (Kuwayama) (Hemiptera: Liviidae) en lima persa. Acta Zoológica Mexicana (n. s.), 31(1): 41-47.
\end{abstract}

RESUMEN. Se evaluó el efecto de seis formulaciones comerciales de insecticidas para el control de los diferentes estados biológicos de Diaphorina citri (Kuwayama) en lima persa en condiciones de invernadero y en laboratorio. Los insecticidas estudiados fueron imidacloprid + betacyflutrin, spirotetramat, imidacloprid, thiametoxam + lambda cyalotrina, imidacloprid + lambda cyalotrina, aceite parafínico de petróleo y un testigo sin aplicación. Se utilizaron especímenes de una cria de D. citri traída de Cuitláhuac, Veracruz en enero de 2012. Se establecieron sobre plantas de lima persa con más de un año de no tener aplicaciones de insecticidas. Los parámetros evaluados fueron toxicidad de los insecticidas en huevecillos, ninfas y adultos. Los ensayos se dispusieron en un diseño completamente al azar, con 10 repeticiones para cada tratamiento, los datos se sometieron a un análisis de varianza (ANOVA) y la prueba de separación de medias por el método de Tukey ( $\mathrm{P}=0.05)$. El aceite parafínico de petróleo causó mortalidad de huevecillos; sin embargo, el imidacloprid retrasó la eclosión de huevecillos respecto a los tratamientos con insecticida. Las ninfas fueron susceptibles a todos los insecticidas evaluados, resultando con mayor mortalidad el imidacloprid e imidacloprid + betacyflutrin. En adultos, las mortalidades fueron superiores que en ninfas. En diferentes fechas después de la aplicación se presentaron mortalidades del 100\% para ambos estados biológicos.

Palabras clave: Insecticidas, huevecillo, ninfa, adulto, Psílido Asiático de los Cítricos.

\section{INTRODUCCIÓN}

La citricultura en México representa una actividad de importancia económica y social para las regiones en donde se establecen estos cultivos. A nivel mundial el país se sitúa en el primer lugar como productor de limas y limones, y en cuarto lugar en la producción de pomelos (FAOSTAT 2013). Sin embargo, la riqueza citrícola se ve amenazada desde el año 2002 por la presencia de Diaphorina citri Kuwayama (Hemíptera: Liviidae) (Burckhardt \& Ouvrard 2012). Según Halbert \& Manjunath (2004) y Bové (2006) la parte más importante del problema es que
Ruiz-Galván, I., Bautista-Martínez, N., Sánchez-Arroyo, H. \& Valenzuela Escoboza, F. A. 2015. Chemical control of Diaphorina citri (Kuwayama) (Hemiptera: Liviidae) in persian lime. Acta Zoológica Mexicana (n. s.), 31(1): 41-47.

ABSTRACT. The effects of six commercial insecticide formulas for the control of the different stages of Diaphorina citri (Kuwayama) on Persian lime were evaluated under greenhouse conditions and in the laboratory. The studied insecticides were: imidacloprid+betacyflutrin, spirotetramat, imidacloprid, thiametoxam+ lambda cyhalothrin, imidacloprid+ lambda cyhalothrin, petroleum paraffin oil (mineral oil), and an untreated control. The specimens used were from a strain of $D$. citri brought from Cuitlahuac, Veracruz in January, 2012. They were established on Persian lime plants that had a year without insecticide applications. The evaluated parameters were insecticide toxicity on eggs, nymphs, and adults. The essays were set up in a totally random design, with 10 replicates for each treatment. The data were subject to a variance analysis (ANOVA) and Tukey's mean separation method $(\mathrm{P}=0.05)$. Petroleum paraffin oil caused mortality in eggs; although, imidacloprid delayed egg emergence more than the other insecticides. Nymphs were susceptible to all the evaluated insecticides, with a higher mortality resulting from imidacloprid and imidacloprid+betacyflut rin. Mortality was greater in adults than in nymphs. At different dates after application, $100 \%$ mortality rates were registered for these two biologic stages.

Key words: Insecticides, egg, nymph, adult, Asian citrus psyllid.

el psílido asiático de los cítricos (PAC), como comúnmente se le conoce, es vector de la bacteria Candidatus Liberibacter asiaticus causante de la enfermedad conocida como Huanglongbing (HLB) o enverdecimiento de los cítricos, la cual se aloja principalmente en el floema de las plantas hospederas (Bové 2006, Garnier et al. 2006, Li et al. 2006).

En la actualidad se le considera como la enfermedad más devastadora para los cítricos en todo el planeta (Halbert \& Nuñez 2004, Bove 2006, Manjunath et al. 2008). Se han reportado cifras superiores a los 63 millones de árboles muertos a causa del HLB principalmente en Asia, 
Sudáfrica y Brasil; lugares donde se presentaron epidemias por esta enfermedad (Bové 2006, Manjunath et al. 2008). Las pérdidas económicas que ha ocasionado la enfermedad van desde reducciones en los rendimientos, hasta la pérdida total de la plantación, esto debido a que los árboles infectados por HLB tienen que eliminarse a fin de evitar la diseminación de la bacteria (Halbert \& Manjunath 2004, Salcedo et al. 2010). En el Continente Americano el enverdecimiento de los cítricos se registró por primera vez en 2004 en Säo Paulo, Brasil (Coletta et al. 2004). En EUA se presentó en 2005 en plantaciones de cítricos en el sur de Florida (Halbert 2005) y en tres años se expandió a todo el estado (FDACS-DPI 2008). En la República Mexicana la enfermedad se detectó en julio de 2009 en huertos de traspatio y comerciales de Yucatán; a cinco años de haberse detectado la enfermedad, ésta se encuentra distribuida en más de la mitad del área citrícola de país. Actualmente el HLB se encuentra en 203 municipios pertenecientes a 13 de los 23 estados citrícolas del país, aunque los mayores daños se registraron en huertas comerciales de Colima, Nayarit, Jalisco, Michoacán y Sinaloa; los síntomas asociados a la enfermedad en las plantas son el moteado asimétrico y caída de hojas, muerte de ramas y deformación de frutos, principalmente (SENASICA 2013). De acuerdo con las proyecciones del Instituto Interamericano de Cooperación para la Agricultura (IICA) en coordinación con el Servicio Nacional de Sanidad, Inocuidad y Calidad Agroalimentaria (SENASICA) el impacto de HLB en México en un lapso de 5 y 8 años, de seguir presente la enfermedad, se tendrá una pérdida de 3 millones de toneladas de fruta equivalente al $41 \%$ de la producción del país (IICA-SENASICA 2010). D. citri es el vector primario de HLB, de este modo es vital dirigir el control hacia este vector como estrategia de control de la enfermedad.

En México el control químico es el más utilizado entre los productores (Tiwari et al. 2011), usan productos de contacto y sistémicos, con acción inmediata y hasta por 30 días (Robles et al. 2011b). Según Alemán et al. (2007), Cortez et al. (2010) y Rogers \& Stansly (2006) las aplicaciones deben iniciarse previo a los máximos picos poblacionales del vector, esto a principios de año cuando las condiciones son favorables para el aumento de la población. Los insecticidas más usados en el mundo para el control del vector, han sido temik, imidacloprid, dimetoato, clorpirifos, malation, cipermetrina, deltametrina, betaciflutrina, spinotetramat, spinetoram, oxamyl, ácidos tricarboxilos, ometoato, sulfoxaflor, aceites minerales, entre otros (Cobelo 2005, Catling 1970, Bové 1986, Dahiya et al. 1994, Sohail et al 2004, Cortez et al. 2010, Villanueva et al. 2011, Orozco \& Cano 2012, Quiñones 2012).
En los últimos años se han propuesto estrategias para controlar a D. citri; sin embargo, el control químico es el método que ha mantenido las poblaciones del insecto más bajas y seguirá usándose a través de los años. Por lo anterior, el objetivo del presente trabajo fue evaluar la susceptibilidad de D. citri a seis insecticidas comerciales.

\section{MATERIALES Y MÉTODOS}

La investigación se realizó en condiciones de invernadero y de laboratorio $\left(27 \pm 3^{\circ} \mathrm{C}\right.$ y $12 \mathrm{~h}$ de fotoperiodo) en Campus Montecillo del Colegio de Postgraduados, Texcoco, Estado de México. Para el estudio se utilizaron brotes provenientes de plantas de lima persa (Citrus latifolia) menores a dos años de edad y con más de un año libres de plaguicidas. Los árboles fueron puestos en bolsas de polietileno negro, con tierra de monte, podados frecuentemente para la generación de brotes, regados con agua potable y fertilizados con urea a dosis de $1 \mathrm{~g} / \mathrm{L}$ de agua, una vez al mes. Las plantas se mantuvieron en jaulas entomológicas de $120 \times 60 \times 60 \mathrm{~cm}$, cubiertas con tela de organza, liberando adultos para la infestación. La colonia del psílido asiático de los cítricos se estableció a partir de colectas realizadas en Cuitláhuac, Veracruz durante los meses de enero a marzo de 2012.

Se evaluaron seis formulaciones comerciales de insecticidas que en el territorio nacional representan una alternativa de control para el PAC. En el Cuadro 1 se incluyen los tratamientos evaluados.

Evaluación en huevecillos. Se utilizó el método propuesto por Ortega-Arenas et al. (1998), con algunas modificaciones. Para cada tratamiento de las plantas infestadas se cortaron 10 brotes de no más de $10 \mathrm{~cm}$ de longitud con huevecillos de entre uno y dos días de edad, el número de huevecillos se contó bajo un microscopio estereoscópico. Posteriormente, se sumergieron en una mezcla de insecticida + agua durante cinco segundos, dejando secar a temperatura ambiente. Cada brote fue colocado en un vaso de plástico desechable de $3.5 \mathrm{~cm}$ de alto y $4.5 \mathrm{~cm}$ de circunferencia, inmerso en un vaso de plástico de $12.5 \mathrm{~cm}$ de alto por $9.5 \mathrm{~cm}$ de circunferencia (que sirvió de jaula), con la parte superior forrada de tela organza. Se dejaron en laboratorio a temperatura ambiente. A los 2, 4 y 6 días después del tratamiento, se evaluó el efecto de los insecticidas para cada caso, contando el número de huevecillos vivos, huevecillos muertos, ninfas vivas, ninfas muertas y adultos (Cuadro 2).

Evaluación en ninfas. En el invernadero se seleccionaron plantas con brotes infestados; se etiquetaron 10 brotes con ninfas de diferentes ínstares, registrando el número de ninfas vivas en cada brote. Los tratamientos fueron asperjados con un atomizador manual hasta "punto de escu- 
Cuadro 1. Tratamientos evaluados en el control químico de Diaphorina citri (Kuwayama) (Hemiptera: Liviidae) en lima persa.

\begin{tabular}{|c|c|c|}
\hline \multicolumn{2}{|r|}{ Tratamiento } & \multirow{2}{*}{$\begin{array}{l}\text { Dosis de producto } \\
\text { comercial formulado }\end{array}$} \\
\hline Nombre comercial & Nombre común & \\
\hline Testigo & Testigo & $400 \mathrm{~L}_{\text {agua }} \mathrm{ha}^{-1}$ \\
\hline Muralla max & imidacloprid + betacyflutrin & $0.4 \mathrm{~L} / 1000 \mathrm{~L}$ de agua \\
\hline Muralla max & imidacloprid + betacyflutrin & 0.5 L/1000 L de agua \\
\hline Movento150 OD & spirotetramat & $0.5 \mathrm{~L} / 1000 \mathrm{~L}$ \\
\hline Confidor 350 SC & imidacloprid & 40 mL/100 L agua \\
\hline Engeo & thiametoxam + lambda cyalotrina & $250 \mathrm{~mL} \mathrm{ha}^{-1}$ \\
\hline Corax SC & imidacloprid + lambda cyalotrina & $300 \mathrm{~mL} \mathrm{ha}^{-1}$ \\
\hline Saf-T-Side & aceite parafínico de petróleo & $1.0 \mathrm{~L} / 100$ de agua \\
\hline
\end{tabular}

Cuadro 2. Toxicidad de insecticidas en huevecillos de Diaphorina citri después de dos, cuatro y seis días después de la aplicación.

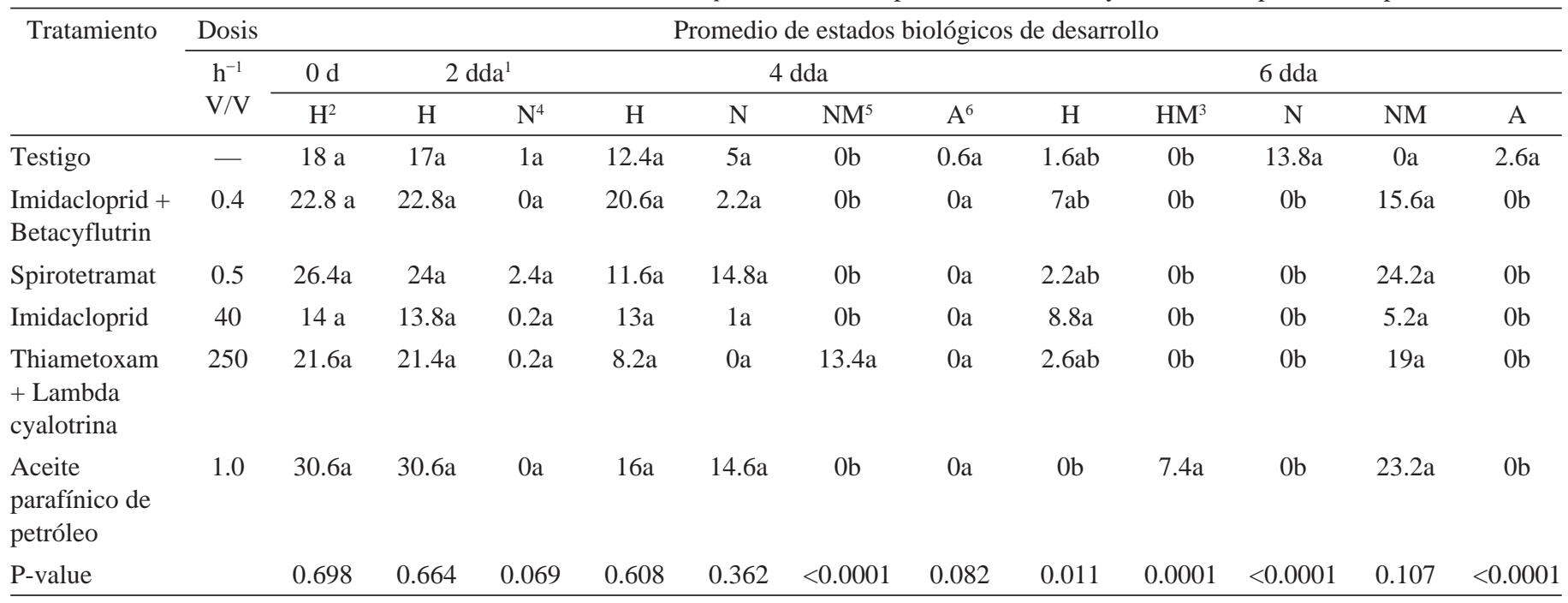

${ }^{1}$ dda: días después de la aplicación. Medias con distinta letra, difieren significativamente, Tukey ( $\left.\alpha \leq 0.05\right)$. ${ }^{2} \mathrm{H}$ : huevos. ${ }^{3} \mathrm{HM}$ : huevos muertos. ${ }^{4} \mathrm{~N}$ : ninfas. ${ }^{5} \mathrm{NM}$ : ninfas muertas. ${ }^{6} \mathrm{~A}$ : adultos.

rrimiento”. Las evaluaciones para registrar el número de ninfas vivas de entre el segundo a cuarto instar se realizaron a los 2 y 7 días después de la aplicación. Para conocer si estaban vivas se removieron con un alfiler entomológico (Cuadro 3).

Evaluación en adultos. Se siguió la metodología señalada por Ortega-Arenas et al. (1998), 10 brotes de cada tratamiento fueron colocados de manera similar a la evaluación en huevecillos. Para el confinamiento de adultos se siguió la técnica usada por Pacheco \& Samaniego (2009) con algunas modificaciones. Se usaron jaulas similares a las usadas en la evaluación de huevecillos, a las cuales se les agregaron dos orificios laterales de $3 \mathrm{~cm}$ de diámetro y forradas con tela organza. Con un aspirador bucal se introdujeron 10 adultos vivos de entre uno y ocho días de edad, evaluando el efecto a las 48 horas.

Análisis estadístico. Todos los ensayos se dispusieron en un diseño completamente al azar, con 10 repeticiones, cada brote fue considerado una repetición. Las compara- ciones de medias se hicieron por el método de Tukey con un nivel de significancia de $\mathrm{P}=0.05$. El análisis se realizó con el paquete estadístico SAS v.9.1.3 (Statistical Analysis System). Previo a las aplicaciones de tratamientos, se llevó a cabo un muestreo de las variables estudiadas para conocer su distribución y corroborar que fueran estadísticamente semejantes.

\section{RESULTADOS Y DISCUSIÓN}

Evaluación en huevecillos. Al iniciar el ensayo cero días (0d) todos los tratamientos con huevecillos no fueron significativamente diferentes. En la primera evaluación dos días después de la aplicación (2dda), en los tratamientos con imidacloprid + betacyflutrin y aceite parafínico de petróleo, no se observó eclosión de huevecillos, mientras que en el resto de tratamientos se registró la presencia de ninfas. A los cuatro días después de la aplicación (4dda), los tratamientos con mayor eclosión de huevecillos fue- 
Cuadro 3. Mortalidad de ninfas de Diaphorina citri a los dos y siete días después de la aplicación de insecticidas.

\begin{tabular}{|c|c|c|c|c|c|}
\hline \multirow{3}{*}{$\begin{array}{ll} & \text { Tratamiento } \\
\text { Testigo } & \end{array}$} & \multirow{3}{*}{$\begin{array}{c}\text { Dosis } \\
400 \mathrm{~L}_{\text {agua }} \mathrm{ha}^{-1}\end{array}$} & \multicolumn{4}{|c|}{ Mortalidad (\% \pm EE) } \\
\hline & & \multicolumn{2}{|c|}{$2 \mathrm{dda}^{1}$} & \multicolumn{2}{|c|}{ 7dda } \\
\hline & & $00 \pm 0.0$ & a & $0 \pm 0.0$ & a \\
\hline Imidacloprid + Betacyflutrin & 0.5 L/1000 L de agua & $100 \pm 0.0$ & b & $100 \pm 0.0$ & b \\
\hline Spirotetramat & $0.5 \mathrm{~L} / 1000 \mathrm{~L}$ & $96.2 \pm 0.4$ & $\mathrm{~b}$ & $98.6 \pm 0.2$ & b \\
\hline Imidacloprid + Lambda cyalotrina & $300 \mathrm{~mL} \mathrm{ha}^{-1}$ & $98.9 \pm 0.2$ & $\mathrm{~b}$ & $100 \pm 0.0$ & $\mathrm{~b}$ \\
\hline Aceite parafínico de petróleo & 1.0 L/100 de agua & $83.2 \pm 0.7$ & b & $87.4 \pm 0.5$ & $\mathrm{~b}$ \\
\hline
\end{tabular}

${ }^{1}$ dda: días después de la aplicación. Medias con distinta letra, difieren significativamente, Tukey $(\alpha \leq 0.05)$.

ron: thiametoxam + lambda cyalotrina, spirotetramat, aceite parafínico de petróleo y el testigo, a diferencia del resto, el testigo presentó los tres estados biológicos vivos; en el tratamiento a base de thiametoxam + lambda cyalotrina las ninfas recién emergidas se registraron muertas. Transcurridos seis días después de la aplicación (6dda) se mostraron diferencias significativas en el número de huevecillos viables, conjuntándose en tres grupos estadísticamente diferentes. El tratamiento con imidacloprid retardó de manera significativa la eclosión de huevecillos en un $62.8 \%$, mientras que los huevecillos tratados con aceite parafínico de petróleo no eclosionaron y se observaron de color oscuro, deshidratados y rotos. En todos los tratamientos las ninfas emergidas murieron excepto en el testigo donde incluso llegaron a la etapa adulta (Cuadro 2).

Este experimento mostró que el aceite parafínico de petróleo tuvo efecto directo en la mortalidad de huevecillos; al respecto Rae et al. (1997) publicó resultados similares indicando que la presencia del PAC se redujo cuando árboles de limón fueron tratados antes de la aparición de adultos, el resultado fue atribuido a la reducción de eclosión de huevecillos por el depósito del aceite de petróleo en los brotes. Según Alemán et al. (2007) y Stadler \& Buteler (2009) indicaron que el mayor efecto de aceites de petróleo se atribuyó a la película de aceite que se forma sobre las formas inmóviles y huevecillos causando asfixia. Por su parte Al Dabel et al. (2008) mencionaron que la pulverización de aceites de petróleo en huevecillos de Ostrinia nubilalis Hubner llegaron a causar mortalidades de 99\%, mientras que en Choristoneura rosaceana (Harris) redujo el número de huevos puestos y la supervivencia (Wins-Purdy et al. 2009). El imidacloprid a los seis días presentó el mayor número de huevecillos sin eclosionar, seguido de imidacloprid + betacyflutrin, thiametoxam + lambda cyalotrina, spirotetramat, estos dos últimos conjuntándose con el testigo en un grupo estadísticamente igual.
Sánchez (2010) documentó que en condiciones normales, la eclosión de huevecillos de $D$. citri se llevó a cabo en tres días, dependiendo del hospedero. En Muralla paniculata el periodo de incubación fue de 3 a 21 días con una viabilidad del 96.1\% (Nava et al. 2007, Tsai \& Liu 2000, Lellani et al. 2012), en nuestro experimento el testigo en lima persa tuvo $92 \%$ de huevos eclosionados, siendo mayor el porcentaje que en el resto de tratamientos. Por consiguiente, la inmersión de brotes en la solución insecticida + agua sólo retardó la eclosión de huevecillos, Raj et al. (2009) reportaron que adultos alimentados en plantas tratadas con imidacloprid, redujo en 33\% el número de huevecillos puestos.

Evaluación en ninfas. En el muestreo preliminar, se consideró una distribución homogénea de los insectos al inicio del experimento ( $p=0.0 .3697$ ). Las ninfas mostraron susceptibilidad en los tratamientos con insecticida. En los muestreos realizados a los dos y siete días después de la aplicación (2dda y 7dda), el rango de mortalidad fue de $83 \%$ a $100 \%$, en ambos casos; los tratamientos con insecticida fueron significativamente diferentes al testigo e iguales entre sí. Al respecto Orozco \& Cano (2012) registraron eficacia del $98 \%$ en el control de ninfas con estos productos. Por su parte Villanueva et al. (2011) y Díaz \& López (2012) encontraron que en arboles de lima persa tratados con imidacloprid + betacyflutrin y thiametoxam + lambda cyalotrina se registró menor infestación de ninfas, encontrando 0.3 ninfas/brote. Al cabo de siete días después de la aplicación (7dda) los tratamientos con imidacloprid resultaron los más eficaces en el control de ninfas de D. citri con $100 \%$ de mortalidad (Cuadro 3). En resultados publicados por Hernández et al. (2012) mencionaron que después de 30 días el imidacloprid ejerce control superior a $88 \%$.

Evaluación en adultos. Al inicio del estudio la distribución de la plaga fue homogénea en todas las unidades experimentales, considerando como media muestral 10 
Cuadro 4. Mortalidad de adultos de Diaphorina citri a los dos días después de la aplicación de insecticidas.

\begin{tabular}{|c|c|c|c|}
\hline \multirow[t]{2}{*}{ Tratamiento } & \multirow[t]{2}{*}{ Dosis } & \multicolumn{2}{|c|}{ Mortalidad $(\% \pm \mathrm{EE})$} \\
\hline & & $2 \mathrm{dda}^{1}$ & \\
\hline Testigo sin aplicación & ------- & $0 \pm 0.0$ & a \\
\hline Imidacloprid + Betacyflutrin & $0.5 \mathrm{~L} / 1000 \mathrm{~L}$ de agua & $100 \pm 0.0$ & b \\
\hline Spirotetramat & $0.5 \mathrm{~L} / 1000 \mathrm{~L}$ & $97.92 \pm 0.2$ & $\mathrm{~b}$ \\
\hline Imidacloprid + Lambda cyalotrina & $300 \mathrm{~mL} \mathrm{ha}^{-1}$ & $100 \pm 0.0$ & $\mathrm{~b}$ \\
\hline Aceite parafínico de petróleo & 1.0 L/100 de agua & $91.5 \pm 0.3$ & b \\
\hline
\end{tabular}

${ }^{1}$ dda: días después de la aplicación. Medias con distinta letra, difieren significativamente, Tukey $(\alpha \leq 0.05)$.

individuos, por lo que se omitió hacer el análisis de varianza inicial. A las 48 horas después de la aplicación, los adultos del PAC mostraron susceptibilidad diferencial significativa en los tratamientos con insecticida (Cuadro 4). La mortalidad fue de $0 \%$ para el testigo, de $91.5 \%$ para el aceite parafínico de petróleo, 97.9\% para thiametoxam + lambda cyalotrina y $97.9 \%$ para spirotetramat; aunque los activos a base de imidacloprid tuvieron mortalidades del 100\% (Cuadro 4). Al respecto Orozco \& Cano (2012) indicaron que tres días después de la aplicación de imidacloprid + bifentrina en limón mexicano, la población se redujo a cero, resultados semejantes reportaron Díaz et al. (2012). Por su parte Pacheco et al. (2012) documentaron que las poblaciones de adultos tratadas con lambdacyhalotrina y zeta-cipermetrina registraron, mortalidades superiores a 99\%, semejante a los resultados obtenidos en esta investigación. Al respecto, Stansly et al. (2009) sugirieron hacer aplicaciones foliares durante el invierno, cuando se encuentran los adultos sobre los huertos.

Diaphorina citri mostró susceptibilidad a los insecticidas probados en sus diferentes estados biológicos, tanto en aspersión directa al follaje, como en la inmersión de brotes. En trabajos de investigaciones recopilados por Cortez et al. (2010) y Robles et al. (2011a) indicaron que los insecticidas de contacto y sistémicos pertenecientes a diferentes grupos toxicológicos, han logrado disminuir las poblaciones de $D$. citri, dependiendo al uso oportuno de cada uno.

Al aceite parafínico de petróleo, con porcentaje de mortalidad alto, lo establecen como una alternativa en un plan de manejo integrado de $D$. citri. Hay que tener en cuenta que en el control químico de este insecto se deben incluir diversos grupos toxicológicos, cuyas dosis y aplicaciones dependerán del estado biológico, grado de infestación y estado fenológico del cultivo. Por otra parte, los largos periodos residuales de los neocotinoides y sus moléculas derivadas que circulan en la planta no los hace particularmente selectivos a la fauna benéfica, aunque su acción sistémica apoya la selectividad de la misma, por lo que pueden considerarse de riesgo medio sobre los enemigos naturales, además de ser considerados como posibles generadores de una alta resistencia.

Agradecimientos. Al Consejo Nacional de Ciencia y Tecnología (CONACYT) por el apoyo económico para el desarrollo de la presente investigación y al Colegio de Postgraduados Institución de Enseñanza e Investigación.

\section{LITERATURA CITADA}

Al Dabel, F., Mensah, R. K. \& Frerot, B. 2008. Effects of nC24 and nC27 petroleum spray oils on oviposition and egg survival of Ostrinia nubilalis Hubner (Lepidoptera, Pyralidae) and Trichogramma brassicae Bezdenko (Hymenoptera, Trichogrammatidae) adults on maize plants. International Journal of Pest Management, 54: 5-11.

Alemán, J., Baños, H. \& Ravelo, J. 2007. Diaphorina citri y la enfermedad Huanglongbing: una combinación destructiva para la producción citrícola. Protección Vegetal, 22: 154-165.

Bové, J. M. 1986. Greening in the Arabian Peninsula. Toward New Techniques for its detection and control. FAO Plant Protection Bulletin, 34: 7-15.

Bové, J. M. 2006. Huanglongbing: A destructive, newly-emerging, century-old disease of citrus. Journal of Plant Pathology, 88: 737.

Burckhardt, D. \& Ouvrard, D. 2012. A revised classification of the jumping plant-lice (Hemiptera: Psylloidea). Zootaxa, 3509: 1-34.

Catling, H. D. 1970. Distribution of the psyllid vectors of citrus Greening disease with notes on the biology and bionomics of Diaphorina citri. FAO Plant Protection Bulletin, 18: 8-15.

Cobelo, L. 2005. Un citrus sin intrusos. Ed. Limusa. D.F. México. $124 \mathrm{p}$.

Coletta, F. H. D., Targon, M. L. P., Takita, M. A., De Negri, J. D., Pompeu Jr., J., Machado, M. A., do Amaral, A. M. \& Muller, G. W. 2004. First report of the causal agent of huanglongbing (“Candidatus Liberibacter asiaticus”) in Brazil. Plant Disease, 88: 1382.

Cortez, M. E., López A., J. I., Hernández, L. M., Fú, A. \& Loera, J. 2010. Control químico de Diaphorina citri Kuwayama en cítricos 
dulces, en México. Selección de insecticidas y época de aplicación. Folleto técnico No. 35 INIFAP-SAGARPA.

Dahiya, K. K., Lakra, K., Dahiya, A. S. \& Singh, S. P. 1994. Bioefficacy of some insecticides against citrus psylla. Diaphorina citri Kuwayama (Hemiptera: Psyllidae). Crop Research (Hisar), 8: $137-140$.

Díaz, Z. U. A. \& López, A. J. I. 2012. Evaluación de mezclas de diferentes activos insecticidas para controlar Diaphorina citri en limón persa. $3^{\circ}$ Simposio Nacional sobre investigación para el manejo del Psílido Asiático de los Cítricos y el Huanglongbing en México. Xalapa, Veracruz, México.

FAOSTAT (Food and Agriculture Organization Corporate Statistical Database). 2013. Food and agricultural commodities production. En sitio web http://faostat.fao.org/ site/339/default.aspx. (Consultado el 26 de abril de 2013).

FDACS-DPI (Florida Department of Agriculture and Consumer Services, Division of Plant Industries). 2008. Huanglongbing (HLB)/Citrus Greening Disease. Disponible en: http://www.doacs. state.fl.us/pi/chrp/greening/citrusgreening.html. (Consultado el 28 de abril de 2013).

Garnier, M., Eveillard, S. J., Cornje, H. F., Le Roux, P. R. \& Bove, J. M. 2006. Genomic characterization of a Liberibacter present in an ornamental rutaceous tree, Calodendrum capense, in the Western Cape province of South Africa. Proposal of 'Candidatus Liberibacter africanus subsp. capensis'. International Journal of Systematic and Evolutionary Microbiology, 50: 2119-2125.

Halbert, S. E. \& Manjunath, K. L. 2004. Asian citrus psyllids (Sternorrhyncha: Psyllidae) and greening disease of citrus: A literature review and assessment of risk in Florida. Florida Entomologist, 87: 330-353.

Halbert S. E. \& Nuñez, K. C. A. 2004. Distribution of the Asian citrus psyllid, Diaphorina citri Kuwayama (Rhynchota:Psyllidae) in the Caribbean basin. Florida Entomologist, 87: 330353.

Halbert, S. E. 2005. Pest Alert: Citrus Greening/Huanglongbing. Florida Department of Agriculture and Consumer Services, Division of Plant Industry. Disponible en: http://www.doacs.state.fl.us/pi/ chrp/greening/citrusgreeningalert.html.

Hernández, F. L. M., Velázquez, M. J. J., López, A. J. I., Urías, L. L. A. \& Márquez, G. J. 2012. Uso de quitosano en mezcla con imidacloprid para el control de ninfas de Diaphorina citri Kuwayama en limón persa (Citrus latifolia Tanaka) $3^{\circ}$ Simposio Nacional sobre investigación para el manejo del Psílido Asiático de los Cítricos y el Huanglongbing en México. Jalapa, Veracruz, México.

IICA-SENASICA. 2010. Evaluación del impacto económico del Huanglongbing (HLB) en la cadena citrícola Mexicana. Disponible en: http://www.iica.int/Esp/regiones/norte/mexico/Publicac iones\%20de\%20la\%20Oficina/B2009e.pdf (Consultado el 28 de abril de 2012).

Lellani, B. H., Aleman, J., Martínez, M. A., Suris, M. \& Ravelo, J. 2012. Ciclo de vida horizontal de Diaphorina citri Kuwayama (Hemiptera: Psilidae) sobre Muralla paniculata L. Revista de Protección Vegetal, 27.

Li, W., Hartung, S. \& Levy, L. 2006. Quantitative real-time PCR for detection and identification of Candidatus Liberibacter species associated with citrus Huanglongbing. Journal of Microbiology Methods, 66: 104-115.

Manjunath, K. L., Halbert, S. E., Ramadugu, S. C., Webb, S. \& Lee, R. F. 2008. Detection of Candidatus Liberibacter asiaticus in Diaphorina citri and its importance in the Management of Ci- trus Huanglongbing in Florida. The American Phytopathological Society, 98: 387-396.

Nava, D. E., Torres, M. L. G., Rodríguez, M. D. L., Bento, J. M. S. \& Parra, J. R. P. 2007. Biology of Diaphorina citri (Hem: Psyllidae) on different hosts and at different temperaturas. Journal of Applied Entomology, 131: 709-715.

Orozco, S. M. \& Cano, H. A. 2012. Evaluación de la efectividad biológica del insecticida imidacloprid + bifenthrin 250/50 para el control del Psílido Asiático (Diaphorina citri) en el cultivo de limón mexicano (Citrus aurantifolia), en Tecomán, Colima. $2^{\circ}$ Simposio nacional sobre el control del Psílido Asiático de los cítricos y el Huanglongbing.

Ortega-Arenas, L. D., Lagunas T., A., Rodríguez M., J. C., Rodríguez H., C., Alatorre R., R. \& Bárcenas O, N. M. 1998. Susceptibilidad a insecticidas en adultos de mosquita blanca Trialeurodes vaporariorum (West.) (Homoptera: Aleyrodidae) de Tepoztlán, Morelos, México. Agrociencia, 32: 249-254.

Pacheco, C. J. J. \& Samaniego, R. J. A. 2009. Método de evaluación de residualidad de insecticidas en adultos de Diaphorina citri Kuwayama (Hemiptera: Psyllidae) $2^{\circ}$ Simposio Nacional sobre investigación para el manejo del Psílido Asiático de los Cítricos y el Huanglongbing en México. Montecillo, México.

Pacheco, C. J. J., Samaniego, R. J. A. \& Fontes, P. A. A. 2012. Tecnología para el manejo integrado del Psílido Diaphorina citri Kuwayama (Hemiptera: Psyllidae) en cítricos en Sonora. Folleto Técnico 88. INIFAP, Sonora, México.

Quiñones, L. S. 2012. Evaluación de campo del Sulfoxaflor, un Nuevo insecticida contra el Psílido Asiático de los cítricos Diaphorina citri Kuwayama (Hemiptera: Psyllidae) en limón mexicano. $2^{\circ}$ Simposio nacional sobre investigación para el manejo del Psílido Asiático de los Cítricos y el Huanglongbing en México. Montecillo, México.

Rae, D. J., Liang, W. G., Watson, D. M., Beattie, G. A. \& Huang, M. D. 1997. Evaluation of petroleum spray oils for control of the Asian citrus psylla, Diaphorina citri (Kuwayama) (Hemiptera: Psyllidae), in China. International Journal of Pest Management, 43: 71-75.

Raj, B. D., Onagbola, O. E., Salyani, M. \& Lukasz, S. L. 2009. Antifeedant and sublethal efects of imidacloprid on Asian Citrus Psyllid, Diaphorina citri. Pest Management Science, 65: 870-877.

Robles, G. M., Velázquez, M. J. J., Manzanilla, R. M. A., Orozco, S. M., Flores, V. R. \& Medina, U.V.M. 2011a. El HLB en árboles de limón mexicano [Citrus aurantifolia (christm) swingle] su dispersión y síntomas en Colima, México. 2 Simposio Nacional sobre investigación para el manejo del psílido asiático de los cítricos y el Huanglongbing en México. Montecillo, México.

Robles, G. M., Velázquez, M. J. J., Manzanilla, R. M. A., Orozco, S. M., Flores, V. R. \& López A., J. I. 2011b. Control químico de Diaphorina citri en limón mexicano. Insecticidas convencionales, productos alternativos y épocas de aplicación. INIFAP Tecomán. Folleto técnico No. 1.

Rogers, M. E. \& Stansly, P. A. 2006. Biology and management of the asian citrus psyllid, Diaphorina citri Kuwayama, in Florida citrus. Bulletin 739. Institute of Food and Agricultural Sciences of the University of Florida. 7 pp.

Salcedo, B. D., Hinojosa, R., Mora, G., Covarrubias, I., DePaolis, F., Cíntora, C. \& Mora, S. 2010. Evaluación del Impacto Económico de Huanlongbing (HLB) en la Cadena Citrícola Mexicana. México: IICA. 124 p.

Sánchez, B. M. 2010. Biología, ecología y control de Diaphorina citri 
Kuwayama (Hemiptara: Psillidae). Tesis de doctorado en ciencias. Colegio de Postgraduados, México. 46 pp.

SENASICA (Servicio Nacional de Sanidad, Inocuidad y Calidad Agroalimentaria). 2013 Acciones Contra el Huanglongbing y su vector en México. Informe marzo Disponible en: http://www. senasica.gob.mx/?doc $=25084$ (Consultado el 28 de abril de 2013).

Sohail, A., Nisar, A. \& Rashad, R. 2004. Studies on Population Dynamics and Chemical Control of Citrus Psylla, Diaphorina citri. International Journal of Agriculture and Biology, 6: 970-973.

Stadler, T. \& Buteler, M. 2009. Modes of entry of petroleum distilled spray-oils into insects: a review. Bulletin of Insectology. Department of Agroenvironmental Sciences and Technologies, 62: 169-169.

Stansly, P., Qureshi, J. \& Arevalo, A. 2009. Why, when and how to monitor and manage Asian citrus psyllid. Citrus Industry, 90: 24-34.
Tiwari, S., Mann, R. S., Rogers, M. E. \& Stelinski, L. L. 2011, Insecticide resistance in field populations of Asian citrus psyllid in Florida. Pest Management Science, 67: 1258-1268.

Tsai, J. H. \& Liu, Y. A. 2000. Biology of Diaphorina citri (Homoptera:Psyllidae) on four host plants. Journal of Economic Entomology, 93: 1721-1725.

Villanueva, J. J. A., Cabrera, M. H., Murillo, C. F. D., Aguilar, R. L., José, P. R. \& Montero, M. A. L. 2011. Insecticidas para el control químico de Diaphorina citri Kuwayama (Hemiptera: Psyllidae) en limón persa en la región central de Veracruz. $2^{\circ}$ Simposio Nacional sobre investigación para el manejo del Psílido Asiático de los Cítricos y el Huanglongbing en México. Montecillo, México.

Wins-Purdy, A. H., Whitehouse, C. G. \& Evenden, M. L. 2009. Effect of Horticultural Oil on Oviposition Behaviour and Egg Survival in the Obliquebanded Leafroller (Lepidoptera: Tortricidae). The Canadian Entomologist, 141: 86-94. 